\title{
Zell- und Gewebsveränderungen durch eine Stosswellenbehandlung
}

\author{
D.F.Hülser, F.Brümmer, J.Brenner, Th.Bräuner, M.Nesper
}

Biologisches Institut, Universität Stuttgart, Abteilung Biophysik

Die extrakorporale Stoßwellenlithotripsie kann seit Beginn ihrer klinischen Anwendung im Jahre 1980 bedeutende Erfolge bei der berührungslosen Nierensteinzertrümmerung vorweisen, was dazu führte, daß sie mittlerweile auch für die Behandlung von Gallensteinen angewendet wird. Da offensichtlich wesentlich geringere Nebenwirkungen als nach Operationen auftreten, hat sich diese Methode weltweit rasch ausgebreitet und es ist nicht verwunderlich, wenn nunmehr auch uber eine Anwendungserweiterung in Richtung Tumortherapie spekuliert wird. Dazu mulssen allerdings die zellulären Auswirkungen der Stoßwellenbehandlung von Geweben genauer charakterisiert werden.

Sto $\beta$ wellen bestehen im typischen Fall nur aus einem einzigen Druckimpuls mit steiler Anstiegsflanke und langsamem Abfall. Somit unterscheiden sie sich eindeutig von Ultraschallwellen, die durch einen sinusförmigen Verlauf aufeinanderfolgender Druck- und Zugperioden charakterisiert sind. Für die klinische Anwendung werden die Stoßwellen in Wasser erzeugt, was eine gute Ankopplung an den Körper zuläßt, da menschliches Gewebe eine dem Wasser vergleichbare akustische Impedanz besitzt. Bei entsprechend sorgfultiger Ankopplung treten daher an der Körperoberfläche keine Reflexionen auf und die Stoßwellen transportieren ihre maximale Energie in das Zielgebiet. Treffen Stoßwellen auf ein Medium mit unterschiedlicher akustischer Impedanz, dann werden sie sowohl beim Ein- als auch beim Austritt reflektiert, was zu Zugwellen fuhrt, die besonders destruktiv wirken. Die außerhalb des Körpers erzeugten Sto $\beta$ wellen (Unterwasserfunkenentladung, elektromagnetischer oder piezoelektrischer Generator, gepulster Laser, Mikroexplosion) können so in den Körper fokussiert werden, da $\beta$ ihre maximale Energie nur in einem begrenzten Fokusbereich zur Verfugung steht. Dennoch treten Zell- und Gewebsveränderungen nach Stoßwellenbehandlung auch außerhalb des Fokusbereichs auf.

Die biologische Wirkung der Stoßwelle ist in unterschiedlichem Zusammenhang beschrieben worden: a) als Nebenwirkungen, die bei einer Behandlung zur Steinzertrummerung am Patienten beobachtet werden, b) als Schäden, die durch Experimente mit Tieren an Zellen oder Geweben im Fokusbereich gefunden wurden und c) als Schäden und Veränderungen an Zellen und Geweben, die in vitro mit Stoßwellen behandelt wurden.

Als Nebenwirkungen treten bei etwa $10 \%$ der Patienten nach Stoßwellenbehandlung petechiale Hauthämatome im Bereich des Eintritts- (7) und des Austrittsfeldes (4) der Stoßwelle auf. Es kann zu perirenalen Hämatomen, verbunden mit subkapsulären Blutungen in den behandelten Nieren kommen (7), die allerdings voll funktionsfähig bleiben, wie Clearance-Tests gezeigt haben (3). Selbst Behandlungen mit 3000 Sto $\beta$ wellen scheinen noch vertretbar zu sein (7). Nach 4 bis 6 Wochen sind in den sto $\beta$ wellenbehandelten Patienten mit Nierenfunktionstests keine Nebenwirkungen mehr feststellbar (8).

Kishimoto und Mitarbeiter (9) haben in physiologischen Untersuchungen 17 verschiedene Substanzen im Blut untersucht und Blutbilder der Patienten vor und nach der Stoßwellenbehandlung erstellt. Leider sind ihre publizierten Daten widersprüchlich und lassen sich nicht eindeutig in eine Dosis- und Zeitabhängigkeit einordnen. Folgt man den Schlußfolgerungen der Autoren, dann erhöht sich nach der Stoßwellenbehandlung das freie Hämoglobin, die Laktatdehydrogenase und die Glutamat-Oxalacetat-Transaminase in dosisabhängiger Weise. Ferner war die Kreatinphosphokinase und der Myoglobingehalt des Blutes einen Tag nach Stoßwellenbehandlung erhöht. Diese Ergebnisse weisen auf eine Hämolyse hin, die von den obengenannten Hämatomen herruhren durfte. Ferner lassen sie den Schluß auf eine gewisse Schädigung von Muskelgewebe $\mathrm{zu}$, was ein direkter, stoßwellenbedingter Effekt sein kann, aber auch einen Sekundäreffekt auf Grund von Durchblutungsstörungen nicht ausschließt.

Neben diesen Befunden an stoßwellenbehandelten Patienten gibt es eine Reihe von Resultaten aus Experimenten, bei denen Organe und Gewebe in Tieren direkt der Stoßwelle ausgesetzt wurden. 
Aus diesen Versuchen mit Hunden $(3,6)$, Schweinen (6), Ratten (4) und Goldhamstern (2) lä $\beta$ t sich zusammenfassend sagen, da $\beta$ in allen gezielt beschossenen Organen (Niere, Galle, Leber, Lunge) Hämatome beobachtet wurden, deren Häufigkeit eher von der zur Erzeugung der Sto $\beta$ welle verwendeten Spannung als von der Anzahl der applizierten Stoßwellen abhing (6). Diese Hämatome sind stets an der Oberfläche der Organe zu finden, größere Blutgefäße weisen keine Schädigung auf, während die Endothelzellschicht der Kapillaren verletzt wurde, was manchmal zu Kapillarverschlüssen führte (2). Da Veränderungen eher in kleinen Gefaßßen oder im Interstitium als im festen Gewebe beobachtet wurden, wird vermutet, daß Kavitationen fur diese Wirkung verantwortlich sein könnten (2).

Es fehit auch nicht an Versuchen, die Stoßwellen zur Behandlung von Tumoren einzusetzen. Russo und Mitarbeiter $(10,11)$ fanden nach invitro-Versuchen eine dosisabhängige verringerte Vitalität und eine reduzierte Klonierungsrate von Mammakarzinom- und Prostatatumorzellen. Berens und Mitarbeiter (1) haben eine höhere Empfindlichkeit gegenuber chemotherapeutisch wirksamen Substanzen bei 3 menschlichen Karzinomazellinien beobachtet.

Diese Ergebnisse scheinen im Widerspruch zu den Resultaten der oben angegebenen in-vivo-Experimente $\mathrm{zu}$ stehen, sind aber durch die unterschiedlichen Versuchsbedingungen $\mathrm{zu}$ erklären. Von beiden Arbeitsgruppen wurden die Zellen als Einzelzell-Suspension im Fokusbereich der Sto $\beta$ welle behandelt, was nicht mit den Behandlungen im Tier zu vergleichen ist. Wir haben uns ebenfalls mit den biologischen und physiologischen Wirkungen der Stoßwellen befaßt, wobei wir besonders darauf geachtet haben, primäre Wirkungen von sekundären Effekten abzugrenzen. Dabei wurden Zellen der Maus Leukämie Linie L1210 als Einzelzell-Suspension behandelt und die epitheloiden Tumorzellen des menschlichen Gebärmutterkarzinoms HeLa sowie die Brusttumorzellen EMT6/Ro der Maus als dreidimensional organisierte Multizell-Sphäroide den Stoßwellen ausgesetzt. Die Zellen wurden jeweils mit Hilfe von Polyethylen-Pipetten in den zweiten Brennpunkt gebracht.

Zur Erzeugung von Stoßwellen benutzten wir einen Experimentallithotripter (Typ XL 1) der Firma Dornier Medizintechnik. Dabei entlädt sich die in einem Hochspannungskondensator gespeicherte Energie (18 kV, $80 \mathrm{nF}$ ) uber eine Unterwasserfunkenstrecke (Elektrode), die im Brennpunkt (F1) eines Messing-Halbellipsoids lokalisiert ist. Durch den Funkenuberschlag zwischen den beiden Polen der Elektrode kommt es zu einer explosionsartigen Verdampfung des umgebenden Wassers und der kugelförmigen Ausbreitung einer Stoßwelle. Aufgrund der geometrischen Eigenschaften des Halbellipsoids werden sämtliche Stoßwellenfronten, die von den Ellipsoidwandungen reflektiert werden, im zweiten Brennpunkt (F2) fokussiert (siehe Fig. 1). Durch die Kreuzung zweier Laserstrahlen, kann F2 exakt angegeben werden.
Um den Einfluß verschiedener Gaskonzentrationen des Lithotripter-Wasserbades $\mathrm{zu}$ untersuchen, brachten wir Kunststeine $(3 \times 3 \times 1,5$ $\mathrm{cm}$ ) aus einer Mischung von Dentalzement und kleinen Glaskügelchen (Dornier) in den zweiten Brennpunkt und setzten diese 125 Stoßwellen aus. In normalem Wasser (Sauerstoffgehalt bei $18^{\circ} \mathrm{C}$ $9,19 \pm 0,16 \mathrm{mg} / 1 ; \mathrm{n}=5$ ) konnte eine weite Verteilung der Einschulsse in die Kunststeine beobachtet werden. In teilweise entgastem Wasser, in dem der Sauerstoffgehalt nur noch $3,86 \pm 0,28 \mathrm{mg} / \mathrm{l}$ $(n=5)$ betrug, trat ein scharfer Fokus mit einem tiefen Krater auf. Aufbrïche des Kunststeins waren auch auf der Austrittsseite der Stoßwelle zu sehen. Dieser erhöhte Schädigungsgrad der Stoßwellen in teilweise entgastem Wasser zeigte sich auch an L1210-Zellsuspensionen: nach einer Behandlung mit 125 Stoßwellen waren in teilweise entgastem Wasser weniger intakte Zellen zu beobachten als in normalem Wasser. Für alle weiteren Experimente wurde die Stoßwellenbehandlung in teilweise entgastem Wasser durchgefuhrt.

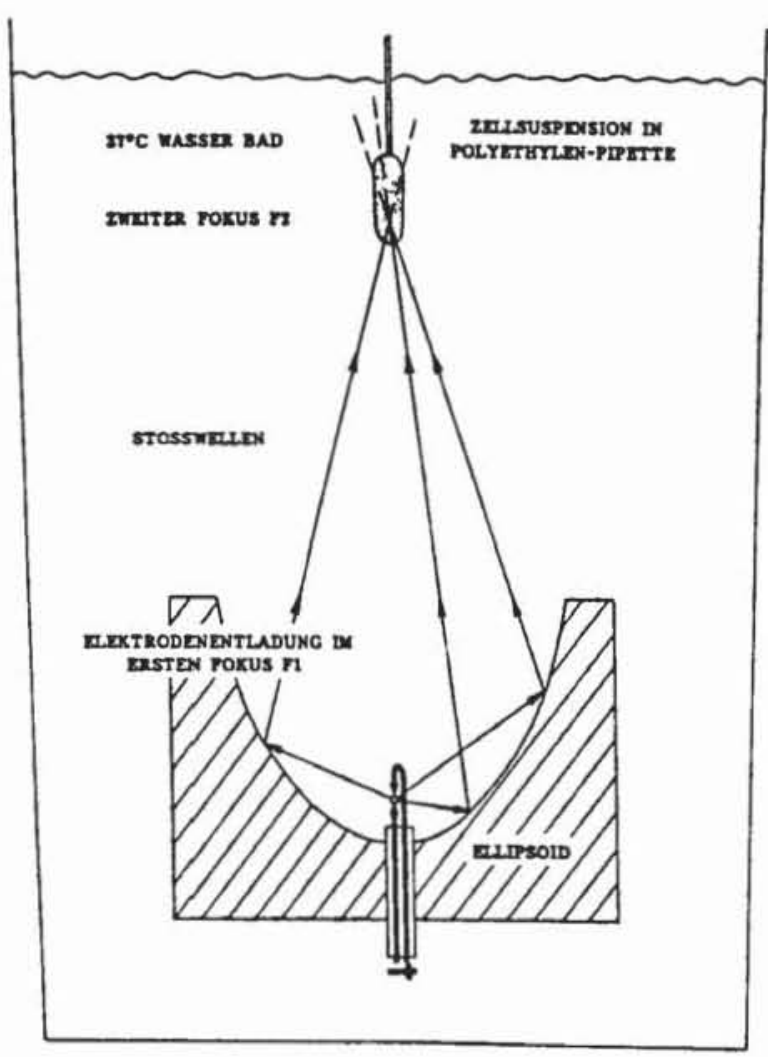

Figur 1: Versuchsaufbau im XL 1 Lithotripter. Die Elektrode für die Unterwasser-Funktenentladung sitzt im Fokus (F1) eines Messing-Halbellipsoids. Die reflektierten Stoßwellen vereinigen sich im zweiten Fokus (F2), wohin die Zellen in Polyethylen-Pipetten eingebracht werden. 
Jede Elektrode zur Stoßwellenerzeugung wurde nur zwischen der 50. und der 1500. Zündung für die Experimente verwendet. Innerhalb dieses Bereichs konnten wir bei Versuchen mit 375 Stoßwellen keine Unterschiede im Grad der Zellschädigung feststellen.

Zellsuspensionen der Maus-Leukämie-Linie $\mathrm{L1210}$, die bei $37^{\circ} \mathrm{C}$ in teilentgastem Wasser mit 500 Stoßwellen behandelt wurden, zeigten im Lichtmikroskop unterschiedliche Schädigungen. Neben unregelmäßigen Zellformen und Änderungen des Färbeverhaltens waren Hohlräume im Zytoplasma zu beobachten. Das Auftreten zahlreicher Zelltrümmer belegte eine völlige Fragmentierung vieler Zellen. Im Elektronenmikroskop zeigten sich Schäden in der Feinstruktur der Mitochondrien, Auftreibungen des endoplasmatischen Retikulums, große Hohlräume im Zytoplasma, eine hohe Elektronentransparenz bei membrangeschädigten Zellen sowie - in unterschiedlichem Ausmaß - eine Trennung des Zytoplasmas vom Zellkern. Unter vergleichbaren Untersuchungsbedingungen beobachteten Russo und Mitarbeiter (11) eine Schädigung der Mitochondrien sowie eine Zunahme an Zellfragmenten. Unsere histologischen Befunde korrelieren mit Bestimmungen der Zellzahl und des physiologischen Zustandes stoßwellen-behandelter Zellen. Zur Quantifizierung des Anteils geschädigter Zellen benutzten wir den Farbstofftest mit Trypanblau. Der Test beruht auf der Tatsache, daß lebende Zellen Trypanblau nicht aufnehmen, während sich bei toten oder geschädigten Zellen sowohl das Zytoplasma als auch der Kern anfärben. Die damit gewonnenen Ergebnisse zeigten keine dosisabhängige Schädigung. Teilweise war bereits in den Kontrollen ein hoher Prozentsatz an Zellen angefärbt. Ähnliche Ergebnisse werden von Russo und Mitarbeitern (10) berichtet. Im Gegensatz dazu ergaben Messungen mit einem elektronischen Zellzählgerät (Coulter Counter) eine deutliche Abnahme intakter Zellen mit steigender Stoßwellenzahl und eine $\mathrm{LD}_{50}$ von 500 Stoßwellen. Die verbleibende Population geometrisch intakter Zellen enthielt jedoch noch eine Subpopulation, die sich mit einem anderen Testverfahren als tote Zellen herausstellte. Dafür wurden die Zellsuspensionen in Fluoresceindiacetat und Propidiumjodid inkubiert und die resultierende Färbung mit einem Durchflußzytometer gemessen. Mit dieser Methode konnten zwischen lebenden - Fluorescein-angefärbten - und toten - Propidiumjodidangefärbten - Zellen unterschieden werden.

Die Ergebnisse erbrachten eine dosis-abhängige Abnahme lebender Zellen. Mit einem Zahlenbeispiel soll dies verdeutlicht werden: Nach 500 Stoßwellen ergab die Coulter Counter Messung 45 \% intakte Zellen. Von diesen 45 \% konnten $80 \%$ als lebend uber die Fluoresceindiacetatfärbung mit dem Durchflußzytometer erkannt werden, d. h. nur 36 \% der Zellen haben die Behandlung mit 500 Stoßwellen überlebt (siehe Fig. 2). Im Posterbeitrag Brümmer, Brenner, Bräuner, Nesper und Hulser sind diese Ergebnisse ausfuhrlich dargestellt.

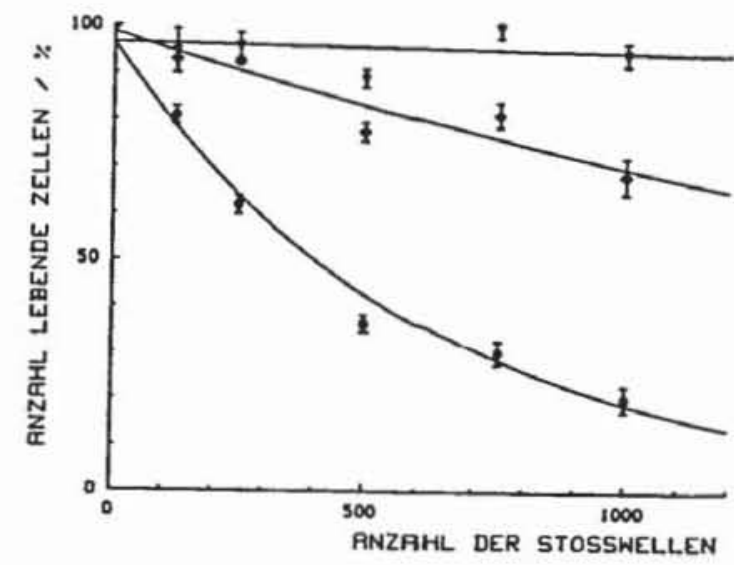

Figur 2: Dosis-Effekt-Kurve stoßwellenbehandelter L1210 Zellen.

- Einzelzellsuspension bei $37^{\circ} \mathrm{C}$;

- Einzelzellsuspension bei $21^{\circ} \mathrm{C}$;

v in Gelatine immobilisierte Zellen bei $21^{\circ} \mathrm{C}$.

Eine stärkere Annäherung an das räumliche Wachstum von Zellen eines Organs ist durch den Einsatz von Multizell-Sphäroiden möglich. Im dreidimensionalen Zellverband eines MultizellSphäroids zeigen die Zellen Organisationsmerkmale und Regulationsleistungen, wie sie vom intakten Gewebe bekannt sind. Suspendierte MultizellSphäroide wurden beim Auftreffen der Stoßwellen stark aufgewirbelt, mit steigender Dosis trubte sich die Suspension durch abgeschlagene Zellen. Bereits nach Behandlung mit 100 Sto $\beta$ wellen konnten in histologischen Schnitten Zellverluste in der äußeren Zellschicht nachgewiesen werden. Mehr Stoßwellen (250-750 Schu $\beta$ ) fuhrten zur Schädigung tieferer Zellschichten, zu Verlusten größerer Zellgruppen im Randbereich des Sphäroids sowie zur völligen Fragmentierung. Diese Schäden hingen von den morphologischen Charakteristika der Tumorzellen und vom Alter der Multizell-Sphäroide ab. Unter gleichen Bedingungen traten bei Multizell-Sphäroiden aus epitheloiden Tumorzellen der Linie HeLa stärkere Schäden auf als bei Multizell-Sphäroiden aus fibroblastoiden Tumorzellen der Linie EMT6/Ro. Ältere Multizell-Sphäroide mit einer Gliederung in eine Randschicht aus vitalen Zellen und ein Zentrum aus nekrotischen Zellen zeigten stärkere Schäden als Multizell-Sphäroide aus vitalen Zellen.

Für das Ausma $\beta$ der Schädigung von suspendierten Multizell-Sphäroiden war die Positionierung des Probenröhrchens von Bedeutung. Deutlich stärkere Schäden waren $\mathrm{zu}$ beobachten, wenn der Boden des Probenröhrchens mit den sedimentierten Multizell-Sphäroiden in den zweiten Brennpunkt F2 gebracht wurde. Während der Behandlung wurden die Multizell-Sphäroide mit jeder einlaufenden Stoßwelle aus dem Sediment 
aufgewirbelt. Eine geringere Schädigung trat dagegen auf, wenn die Mitte des ca. $4 \mathrm{~cm}$ langen Probenröhrchens in den zweiten Brennpunkt F2 positioniert wurde und somit das Sediment ca $2 \mathrm{~cm}$ unterhalb von F2 (in Richtung F1) lag. In diesem Fall blieb die heftige Aufwirbelung der Multizell-Sphäroide beim Einlaufen der Sto $\beta$ welle aus. Elektronenmikroskopische Untersuchungen sto $\beta-$ wellen-behandelter Multizell-Sphäroide ergaben ähnliche Befunde wie sie für Einzelzellsuspensionen der Linie L1210 beschrieben wurden. Besonders starke Zellschädigungen und -verluste waren in den Randschichten $\mathrm{zu}$ beobachten.

Dieses Aufwirbeln der Suspensionen aus Einzelzellen oder aus Multizell-Sphäroiden zeigt, daß sekundäre Schädigungen durch Beschleunigungen und Scherkräfte nicht ausgeschlossen werden können. Wir haben daher die Zellen mit Ficoll und Alginat immobilisiert. Beide Substanzen erbrachten zwar eine hohe Viskosität, doch war stets eine Zunahme toter Zellen bereits in den Kontrollen zu beobachten. Dieser Umstand machte Ficoll und Alginat für diese Art der Immobilisierung unbrauchbar. Dagegen stelle sich eine $12 \%$-ige Gelatinelösung in Medium als sehr vorteilhaft heraus: sie hat nicht nur eine ähnliche akustische Impedanz wie tierische Gewebe, sondern sie ist bei $37^{\circ} \mathrm{C}$ flüssig und erstarrt bei niedrigen Temperaturen. Dies erlaubt die Aufnahme der L1210-Zellsuspension in flussiger Gelatine und eine Stoßwellenbehandlung bei $21^{\circ} \mathrm{C}$ in verfestigter Gelatine. Durch Erwärmen auf $37^{\circ} \mathrm{C}$ kann die Zellsuspenson wiedergewonnen werden und die Messungen mit dem Coulter Counter bzw. dem Durchflußzytometer durchgeführt werden. Wie Figur 2 zeigt, war nach einer Stoßwellenbehandlung immobilisierter L1210-Zellen keine dosisabhängige Schädigung feststellbar. Dies zeigt deutlich, daß andere Mechanismen als das Durchlaufen von Stoßwellen für den hohen Grad der Zellschädigung an Zellsuspensionen verantwortlich sein müssen.

In weiteren Experimenten wurden auch die Multizell-Sphäroide durch Einschluß in eine 2-3 \%-ige Agarlösung (in physiologischer Pufferlösung) bzw. in eine $12 \%$-ige Gelatinelösung (in Kulturmedium) im Probengefä $\beta$ immobilisiert. Diese Agar- oder Gelatinematrix verhinderte die starke Beschleunigung, der die Multizell-Sphäroide in Suspension beim Durchlaufen der Stoßwellen ausgesetzt waren. Unter diesen Bedingungen war sowohl in der licht- als auch in der elektronenmikroskopischen Histologie auch nach Behandlung mit 500-750 Stoßwellen keine Schädigung der Multizell-Sphäroide zu erkennen. Diese Befunde sind im Posterbeitrag Bräuner, Brümmer und Hülser ausfuhrlich dargestellt.

Unsere Ergebnisse erklären auch, warum Russo und Mitarbeiter (11) nach Stoßwellen-Behandlung von Ratten mit transplantierten Prostatakarzinomen keine Schädigung dieser Tumorzellen beobachteten, während dieselben Zellen als Einzelzellsuspensionen fragmentiert wurden.

Wir schließen aus unseren vergleichenden Experimenten mit suspendierten und immobilisierten Einzelzellen und Multizell-Sphäroiden, daß
Sekundäreffekte der Stoßwellen für die Schädigung suspendierter Zellen verantwortlich sind. In Flüssigkeiten verursachen die Stoßwellen Kavitationen und Jetströme, was zur heftigen Durchwirbelung der suspendierten Zellen fuhrt. Die damit verbundenen hohen Beschleunigungen und starken Scherkräfte lassen die Zellen aufbrechen. Die Immobilisierung von Zellen in Agar oder Gelatine schlie $\beta$ t dagegen jede Beschleunigung und damit zusammenhängende Sekundäreffekte aus und schafft damit Bedingungen wie sie für Zellen im Verband eines Gewebes oder Organs im Menschen oder Tier anzutreffen sind. Dies erklärt auch, warum in vivo Zellschäden nur in stark vaskularisierten Organen oder Geweben beobachtet wurden.

Unsere Arbeiten wurden vom BMFT und von Dornier Medizintechnik unterstultzt.

\section{L i t e r a t u r}

1.) Berens, M. E. (1987), persönliche Mitteilung

2.) Brendel, W.; Delius, M. und Goetz, A. (1987) Effect of shock waves on the microvasculature. Prog. appl. Microcirc. 12, 41-50.

3.) Chaussy, Ch.; Schmiedt, E.; Jocham, D.; Schuller, J.; Brandl, H.; und Liedl, B. (1984) Extracorporeal Shock-Wave Lithotripsy (ESWL) for Treatment of Urolithiasis Urology 23, 59-66.

4.) Chaussy, Ch.; Schmiedt, E.; Jocham, D.; Fuchs, G.; Brendel, W.; Forssmann, B. und Hepp, W. (1986c) Extracorporeal Shock Wave Lithotripsy (Edited by Ch. Chaussy), Karger, Basel.

5.) Delius, M.; Enders, G.; Heine, G.; Stark, J.; Remberger, K. und Brendel, W. (1987) Biological effects of shock waves: lung hemorrhage by shock waves in dogs - pressure dependence. Ultrasound in Med. \& Bịol. 13, 61-67.

6.) Delius, M.; Enders, G.; Xuan, Z.; Liebich, H.-G. und Brendel, W. (1988) Biological effects of shock waves: kidney damage by shock waves in dogs - dose dependence. Ultrasound in Med. \& Biol. 14, 117-122.

7.) Eisenberger, F. und Rassweiler, J. (1986) Extrakorporale Stoßwellenlithotripsie im Wandel. Akt. Urol. 17, 229-233.

8.) Kaude, J. V.; Williams, C. M.; Millner, M. R.; Scott, K. N. und Finlayson, B. (1985) Renal Morphology and Function Immediately after Extracorporeal Shock-Wave Lithotripsy. Am. J. Roentg. 145, 305-313.

9.) Kishimoto, T.; Yamamoto, K.; Sugimoto, T.; Yoshihara, H. und Maekawa, M. (1986) Side Effects of Extracorporeal Shock-Wave Exposure in Patients Treated by Extracorporeal Shock-Wave Lithotripsy for Upper Urinary Tract Stone. Eur. Urol. 12, 308-313.

10.) Russo, P.; Stephenson, R. A.; Mies, C.; Huryk, R.; Heston, W. D. W.; Melamed, M. R.; und Fair, W. R. (1986) High energy shock waves suppress tumor growth in vitro and in vivo. J. Urol. 135, 626-628.

11.) Russo, P.; Mies, C.; Huryk, R.; Heston, W. D. W. und Fair, W. R. (1987) Histopathologic and ultrastructural correlates of tumor growth suppression by high energy shock waves. J. Urol. 137, 338-341. 